\title{
Correction to: A case of primary distal-type epithelioid sarcoma of the lumbar vertebra with a review of literature
}

\author{
Ayako Ura ${ }^{1} \cdot$ Tsuyoshi Saito $^{1}$ (D) Toru Motoi $^{2} \cdot$ Tatsuya Takagi $^{3} \cdot$ Yoshiyuki Suehara $^{3} \cdot$ Taisei Kurihara $^{1,3} \cdot$ Kei Sano $^{1,3}$. \\ Keita Sasa ${ }^{1,3} \cdot$ Takuo Hayashi $^{1} \cdot$ Takashi Yao $^{1}$
}

Published online: 18 November 2020

(C) Springer-Verlag GmbH Germany, part of Springer Nature 2020

\section{Correction to: Mycorrhiza \\ https://doi.org/10.1007/s00428-020-02955-w}

The original published version of the above article contained an error. Under the section "Pathological findings", the text originally read, "One of the four microsatellite markers (SMARCB1 301) showed loss of heterozygosity (LOH) in the tumor sample (Fig. 6)." should be read as, "One of the four microsatellite markers (SMARCB1 303) showed loss of heterozygosity (LOH) in the tumor sample (Fig. 6). The original article has been corrected.

Publisher's note Springer Nature remains neutral with regard to jurisdictional claims in published maps and institutional affiliations.

The online version of the original article can be found at https://doi.org/ $10.1007 / \mathrm{s} 00428-020-02955-\mathrm{w}$

Tsuyoshi Saito

tysaitou@juntendo.ac.jp

1 Department of Human Pathology, School of Medicine, Juntendo University, 2-1-1, Hongo, Bunkyo-ku, Tokyo 113-8421, Japan

2 Department of Diagnostic Pathology, Tokyo Metropolitan Cancer and Infectious Disease Center Komagome Hospital, 3-18-22, Honkomagome, Bunkyo-ku, Tokyo 113-8677, Japan

3 Department of Orthopaedic Surgery, School of Medicine, Juntendo University, 2-1-1, Hongo, Bunkyo-ku, Tokyo 113-8421, Japan 